\title{
ASXL1 frameshift mutations drive inferior outcomes in CMML without negative impact in MDS
}

\author{
David A Sallman', Rami Komrokji', Thomas Cluzeau ${ }^{2,3}$, Christine Vaupel ${ }^{4}$, Najla H Al Ali', Jeffrey Lancet ${ }^{1}$, Jeff Hall ${ }^{4}$, \\ Alan List ${ }^{1}$, Eric Padron ${ }^{1}$ and Jinming Song ${ }^{5}$
}

Next-generation sequencing (NGS) has revolutionized the diagnostic, prognostic and treatment paradigms in myeloid malignancies. Although somatic mutations can be identified in the majority of patients with myelodysplastic syndromes (MDS) and chronic myelomonocytic leukemia (CMML), the mutational spectrum and prognostic significance of individual mutations is dependent on disease subtype and remains incompletely understood ${ }^{1,2}$. In CMML, mutations in the addition of sex combs-like 1 (ASXL1) gene are the only mutations consistently associated with inferior survival in multivariable analysis and as such refine discrimination of clinical prognostic mod$\mathrm{els}^{3,4}$. Nonetheless, these studies also suggest that the type of mutation may have prognostic significance as ASXL1 missense mutations are not similarly predictive of clinical outcomes ${ }^{5}$. Additional studies have confirmed the prognostic significance of $A S X L 1$ mutations in CMML while also identifying mutations of $C B L, R U N X 1, N R A S$, and $S E T B P 1$ to have prognostic relevance ${ }^{6}$. In MDS, mutations involving TP53, EZH2, ETV6, RUNX1, and ASXL1 genes are associated with inferior survival, albeit $A S X L 1$ mutations are of only borderline significance ${ }^{7,8}$. Importantly, the prognostic relevance of type of $A S X L 1$ mutation has not been analyzed in MDS patients. These data suggest that there are distinct disease-specific implications in MDS versus CMML that apply to a similar mutational spectrum. Given the predicted differences in prognostic significance of ASXL1 between MDS and CMML, we sought to compare the impact of $A S X L 1$

\footnotetext{
Correspondence: David A Sallman (david.sallman@moffitt.org)

${ }^{1}$ Malignant Hematology Department, H. Lee Moffitt Cancer Center and

Research Institute, Tampa, FL 36612, USA

${ }^{2}$ Cote D'azur University, Nice Sophia Antipolis University, Hematology

Department, CHU Nice, Nice 06200, France

Full list of author information is available at the end of the article
}

mutations in a combined cohort comprised of both diseases. We identified that although $A S X L 1$ mutation was predictive for outcome in CMML (HR 2.97, 95\% CI $1.21-7.06 ; P=0.02)$, it had no impact on outcome in MDS patients (HR 1.04, $P=0.87$ ). In addition, whereas the negative significance of $A S X L 1$ mutation status was dependent on frameshift (FS) mutations (HR 3.85, 95\% CI 1.84-15.61, $P=0.0026)$ in CMML, the type of mutation had no impact on MDS prognosis even when accounting for missense mutations.

Patients were identified retrospectively from the Moffitt Cancer Center (MCC) database that had NGS performed and a diagnosis of CMML or MDS according to WHO criteria (including patients with refractory anemia with excess blasts in transformation according to FAB). Pathology review was performed at MCC. This study was approved by the MCC Scientific Review Committee and institutional review board. From May 2013 to July 2015, a total of $60 \mathrm{CMML}$ patients were identified who underwent NGS who were compared to a cohort of 195 MDS patients (Table 1). From May 2013 to October 2014, targeted amplicon based NGS of 21 myeloid genes was performed on DNA extracted from mononuclear cells of $\mathrm{BM}$ aspirate or peripheral blood as previously described, which was followed by NGS using a 32 gene panel ${ }^{9}$. The lower limit of detection for variant calling was set at a 5\% variant allele frequency (VAF) and the minimum depth of coverage was $500 \times$. Clinical characteristics were cataloged from the date of mutation analysis. Kaplan-Meier estimates were used to estimate OS and analyzed from the date of mutation identification. Cox regression models were cr7eated to adjust for clinical characteristics. Categorical and continuous variables were compared by Fisher's exact test and Mann-Whitney's test, respectively. 
Table 1 Baseline characteristics of the study population by ASXL1 mutation status (frameshift and nonsense)

\begin{tabular}{|c|c|c|c|c|}
\hline & \multicolumn{2}{|c|}{ MDS cohort } & \multicolumn{2}{|c|}{ CMML cohort } \\
\hline & $\begin{array}{l}\text { ASXL1 MT } \\
n=36\end{array}$ & $\begin{array}{l}\text { ASXL1 WT } \\
n=159\end{array}$ & $\begin{array}{l}\text { ASXL1 MT } \\
n=28\end{array}$ & $\begin{array}{l}\text { ASXL1 WT } \\
n=32\end{array}$ \\
\hline Median age (years) & $74(51-92)$ & $72(34-100)$ & $74(48-88)$ & $74(57-94)$ \\
\hline Male & $25(69 \%)$ & 99 (62\%) & $21(75 \%)$ & $20(63 \%)$ \\
\hline Female & $11(31 \%)$ & $60(38 \%)$ & $7(25 \%)$ & $12(37 \%)$ \\
\hline $\begin{array}{l}\text { Median } \\
\text { hemoglobin }(\mathrm{g} / \mathrm{dl})\end{array}$ & 9.1 & 9.4 & 10.1 & 10.9 \\
\hline $\begin{array}{l}\text { Median platelets } \\
(\mathrm{G} / \mathrm{L})\end{array}$ & 65 & 90 & 92 & 75 \\
\hline Median ANC & 1.32 & 1.42 & 7.87 & 5.67 \\
\hline $\begin{array}{l}\text { Median monocyte } \\
\text { count }\end{array}$ & 0.22 & 0.24 & 1.96 & 2.58 \\
\hline $\begin{array}{l}\text { Median BM Blast \% } \\
\text { IPSS }\end{array}$ & 4 & 4 & 4 & 4 \\
\hline Low & $5(14 \%)$ & $40(25 \%)$ & $12(43 \%)$ & $15(47 \%)$ \\
\hline Intermediate 1 & $17(47 \%)$ & $50(31 \%)$ & $10(36 \%)$ & $13(41 \%)$ \\
\hline Intermediate 2 & $3(8 \%)$ & $37(23 \%)$ & $3(11 \%)$ & $4(12 \%)$ \\
\hline High & $9(25 \%)$ & $32(20 \%)$ & $3(11 \%)$ & $0(0 \%)$ \\
\hline $\begin{array}{l}\text { \# of mutations } \\
\text { (median) }\end{array}$ & $2^{*}$ & 1 & 3 & 2 \\
\hline ASXL1 Mutation ${ }^{a}$ & & & & \\
\hline $\begin{array}{c}\text { ASXL1 } \\
\text { c.1934dupG }\end{array}$ & $7(19 \%)$ & & $8(29 \%)$ & \\
\hline Frameshift & $25(69 \%)$ & & $18(64 \%)$ & \\
\hline Nonsense & $11(31 \%)$ & & $10(36 \%)$ & \\
\hline HMA treatment & $25(69 \%)$ & 89 (56\%) & $16(57 \%)$ & $15(47 \%)$ \\
\hline Allo-HSCT & $7(19 \%)$ & $23(15 \%)$ & $3(11 \%)$ & $4(13 \%)$ \\
\hline $\begin{array}{l}\text { Median OS } \\
\text { (months) }\end{array}$ & 15.5 & 17.9 & $11.9^{* *}$ & NR \\
\hline
\end{tabular}

MDS myelodysplastic syndrome, CMML chronic myelomonocytic leukemia, $M T$ mutant, WT, wild type, ANC absolute neutrophil count, BM bone marrow, IPSS international prognostic scoring system, HMA hypomethylating agent, allo-HSCT allogeneic hematopoetic stem cell transplantation, OS overall survival ${ }^{*} P<0.0001 ;{ }^{*} P=0.02$

${ }^{a}$ ASXL1 missense mutations occurred in $29 \%(n=15)$ and $7 \%(n=2)$ of the MDS and CMML cohorts, respectively

All tests were two sided with statistically significant variables set at $P<.05$.

Overall, ASXL1 mutation was identified in $26 \%(n=51)$ and $50 \%(n=30)$ of MDS and CMML patients, respectively $(P=0.0008)$. Although the distribution of FS and nonsense (NS) mutations were similar between the MDS and CMML cohorts (Table 1), missense mutations represented only $7 \%(n=2)$ of $A S X L 1$ mutations in the
CMML cohort compared to $29 \%(n=15)$ in the MDS cohort $(P=0.02)$. In patients with $A S X L 1$ FS and NS mutations, there were no significant differences compared to wildtype patients in the CMML or MDS subgroups with regards to cytopenias, monocyte count, bone marrow blasts or IPSS risk classification (Table 1). However, MDS patients with ASXL1 mutations had a significant increased absolute number of gene mutations (median 2 versus $1, P$ $<0.0001$ ), which approached significance in the CMML cohort (median 3 versus $2, P=0.08$ ). In regards to therapeutic intervention, there was no difference in utilization of hypomethylating agent therapy or allogeneic hematopoietic cell transplantation.

We first evaluated the impact of $A S X L 1$ mutation on survival in the CMML cohort given prior data that found mutation to be predictive of inferior OS, albeit only when FS and NS mutations were included ${ }^{4}$. Indeed, CMML patients with $A S X L 1$ FS or NS mutations had inferior OS with a median OS of 11.9 months versus NR in WT patients (Fig. 1a, HR 2.97, 95\% CI 1.21-7.06; $P=0.02$ ). Of interest, type of mutation was predictive in the CMML cohort with a median OS of 9.9 months in ASXL1 FS mutant patients versus not reached (NR) in NS or wildtype patients (Fig. $1 \mathrm{~b}, P=0.01$ ). In comparison to WT patients, ASXL1 FS patients had significantly inferior OS (median OS 9.9 months vs NR; $P=0.0026$ ) while NS mutant patients had no difference in survival $(P=0.70)$. In multivariable analysis incorporating age, sex, and IPSS, $A S X L 1$ FS mutation had the greatest impact on OS (HR 5.87, 95\% CI 1.98-17.4, $P=0.001)$. The clonal burden as defined by variant allele frequency of ASXL1 FS or NS mutations did not further stratify survival in the CMML cohort. In contrast to the CMML cohort, ASXL1 mutation had no impact on outcome in the MDS cohort with a median OS of 15.5 months versus 17.9 months in wildtype patients (Fig. 1c, HR 1.04, $P=0.87$ ). Furthermore, type of mutation had no effect on survival with a median OS of 14.3 months with FS, 16.0 months with NS and 18.6 months with missense mutations (Fig. $1 \mathrm{~d}, P=0.95$ ). Exclusion of $A S X L 1$ missense mutations had no impact on the prognostic impact of $A S X L 1$ mutations (median OS of 14.3 months FS/NS patients versus NR in wildtype patients; HR $1.18, P=0.42)$. Subgroup analysis of $A S X L 1$ mutant MDS patients without excess blasts (i.e., $<5 \%$ ) identified a trend for inferior OS in the mutant cohort (median OS 15.5 months versus NR in the wildtype cohort; Fig. 1e, HR 1.85; $P=0.08$ ). However, type of ASXL1 mutation $(P=0.14)$ or restriction of missense mutations $(P=0.23)$ did not further stratify outcomes in this patient population. In contrast in patients with excess blasts, median OS was 12.5 months in ASXL1 mutant patients versus 8.7 months in wildtype patients although not statistically significant (Fig. 1f, HR 0.68; $P=0.23$ ). 


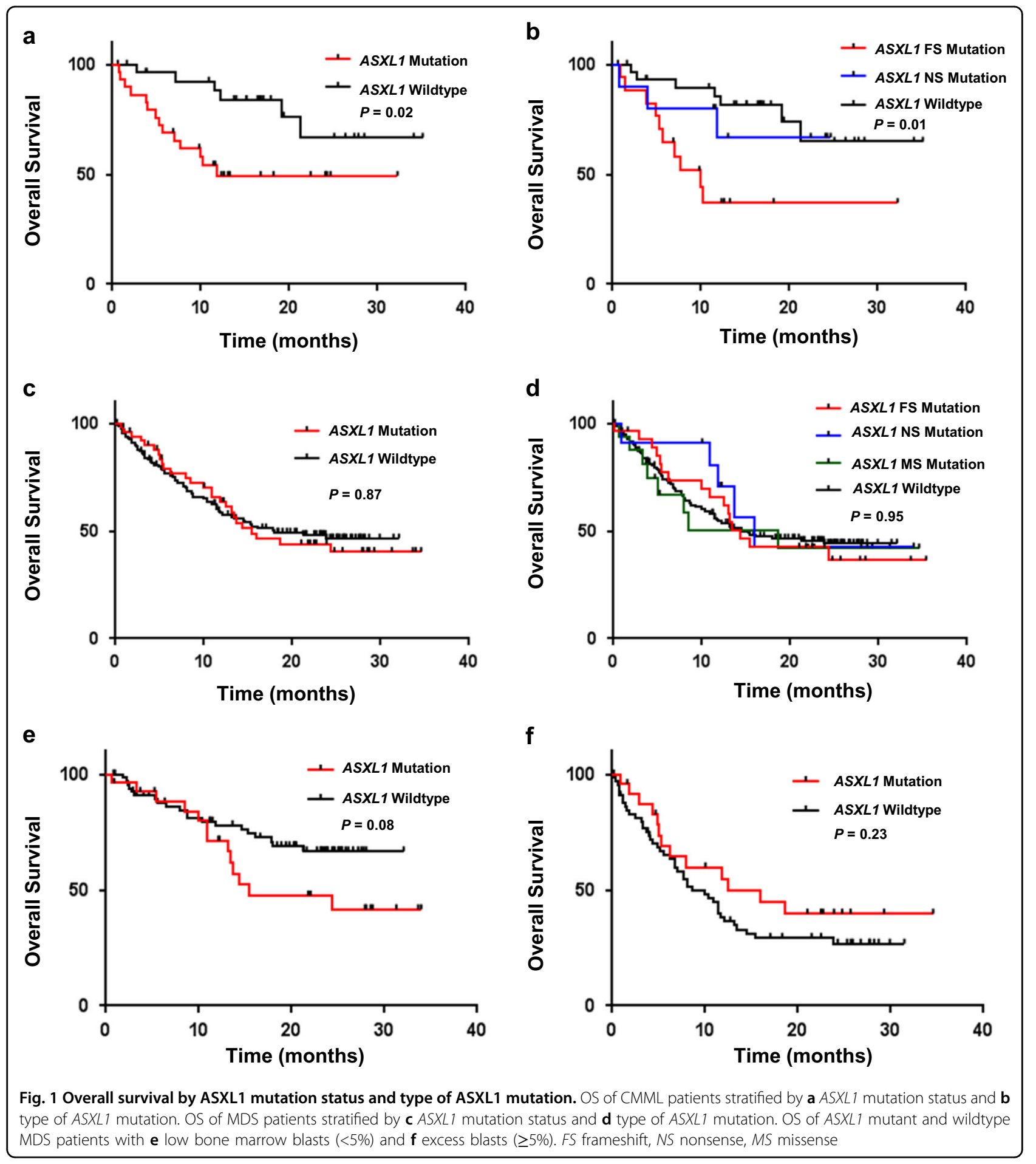

We next evaluated differences between the molecular architecture of ASXL1 mutant cases. CMML patients with ASXL1 mutation had significantly increased cooccurrence of SRSF2 (15.7\% versus $5 \%, P=0.01)$ as well as higher risk mutations including $R U N X 1$ (9.8\% versus $3.6 \%$; $P=0.04), C B L(7.8 \%$ versus $1.2 \%, P=0.02)$ and
EZH2 (9.8\% versus $0.8 \%, P=0.0001)$. In addition, $A S X L 1 /$ TET2 co-mutation was significantly more common in CMML patients $(30.8 \%$ versus $7.2 \%, P=0.0001)$ with no statistically significant co-mutation in the MDS subgroup. ASXL1 mutations in myeloid malignancies have been shown to be loss-of-function mutations resulting in 
myeloid transformation via loss of polycomb repressive complex 2 (PRC2)-mediated H3K27 tri-methylation ${ }^{10}$. Furthermore, leukemogenesis in ASXL1-deficient cells occurs through cooperation with secondary mutations. Additionally, knockdown of ASXL1 impairs granulomonocytic differentiation and leads to overexpression of PRC2 targets ${ }^{11}$. Patnaik and colleagues have evaluated the prognostic interplay of $A S X L 1 / T E T 2$ mutation status in CMML and identified TET2 mutant patients without ASXL1 mutations to have improved OS in comparison to co-mutant patients which had the shortest survival ${ }^{12}$. Perhaps, critical to the prognostic relevance of ASXL1 mutation is its collaboration with other mutations. To this regard, we highlight a significant correlation of higher risk mutations as well as the ASXL1/TET2 co-mutant genotype in CMML compared to MDS patients.

Recent serial molecular annotation of ASXL1 mutant MDS patients showed that the ASXL1 clone was unchanged in all cases at the time of disease progression $(n=32)$ and was only predictive of survival when analysis was restricted to lower risk patients ${ }^{13}$. Our data support these findings as there was a trend for inferior OS in MDS patients without excess blasts. Nazha and colleagues recently created and validated a new molecular prognostic model by incorporating molecular data with IPSS-R risk categorization on a total of $429 \mathrm{MDS}$ and $79 \mathrm{CMML}$ patients where ASXL1 mutation was not predictive of $\mathrm{OS}^{14}$. Altogether, these data suggest that although ASXL1 mutations play a key role in the pathogenesis of MDS, they do not have prognostic relevance in higher risk populations. In contrast, our data further validate the prognostic role of ASXL1 mutations in CMML. Although previous studies confirmed that missense mutations do not impact outcomes in CMML, our study suggests that FS mutations are the most prognostically relevant. Understanding the functional consequence on protein function based on type of $A S X L 1$ mutation needs to be evaluated in future study.

In conclusion, we have identified that only ASXL1 FS mutations predict inferior survival in CMML while ASXL1 mutation status was not predictive of inferior outcomes in the total cohort of MDS patients, regardless of type of mutation. Notably, the differential prognostic impact of ASXL1 mutations in CMML versus MDS patients could be dependent on disease-specific secondary mutations that co-occur with $A S X L 1$ mutations. Furthermore, prognostic relevance of ASXL1 mutations in MDS patients appears to be restricted to lower risk disease. Together, this study highlights significant heterogeneity in the prognostic relevance of ASXL1 mutations in MDS and CMML.

\section{Acknowledgements}

This work was supported by the Edward P. Evans Foundation to DAS.

\section{Author details}

'Malignant Hematology Department, H. Lee Moffitt Cancer Center and Research Institute, Tampa, FL 36612, USA. ${ }^{2}$ Cote D'azur University, Nice Sophia Antipolis University, Hematology Department, CHU Nice, Nice 06200, France. ${ }^{3}$ INSERM U1065, Mediterranean center of molecular medicine, Nice 06200, France. ${ }^{4}$ Navigate BioPharma Services, Inc., a Novartis company, Carlsbad, CA 92008, USA. ${ }^{5}$ Hematopathology and Laboratory Medicine Department, H. Lee Moffitt Cancer Center and Research Institute, Tampa, FL 36612, USA

\section{Competing interests}

$\mathrm{CV}$ and $\mathrm{JH}$ are employees of Navigate BioPharma, a Novartis company.

\section{Publisher's note}

Springer Nature remains neutral with regard to jurisdictional claims in published maps and institutional affiliations.

Received: 17 August 2017 Accepted: 23 August 2017

Published online: 27 November 2017

\section{References}

1. Papaemmanuil, E. et al. Clinical and biological implications of driver mutations in myelodysplastic syndromes. Blood 122, 3616-3627; quiz 3699 (2013).

2. Malcovati, L. et al. Driver somatic mutations identify distinct disease entities within myeloid neoplasms with myelodysplasia. Blood 124, 1513-1521 (2014).

3. Patnaik, M. M. et al. ASXL1 and SETBP1 mutations and their prognostic contribution in chronic myelomonocytic leukemia: a two-center study of 466 patients. Leukemia 28, 2206-2212 (2014).

4. Itzykson, R. et al. Prognostic score including gene mutations in chronic myelomonocytic leukemia. J. Clin. Oncol. 31, 2428-2436 (2013).

5. Patnaik, M. M. et al. Mayo prognostic model for WHO-defined chronic myelomonocytic leukemia: ASXL1 and spliceosome component mutations and outcomes. Leukemia 27, 1504-1510 (2013).

6. Padron, E. et al. An international data set for CMML validates prognostic scoring systems and demonstrates a need for novel prognostication strategies. Blood Cancer J. 5, e333 (2015).

7. Bejar, R. et al. Somatic mutations in MDS patients are associated with clinical features and predict prognosis independent of the IPSS-R: analysis of combined datasets from the International Working Group for Prognosis in MDSMolecular Committee. Blood 126, 907-907 (2015).

8. Bejar, R. et al. Clinical effect of point mutations in myelodysplastic syndromes. N. Engl. J. Med. 364, 2496-2506 (2011).

9. Sallman, D. A. et al. Impact of TP53 mutation variant allele frequency on phenotype and outcomes in myelodysplastic syndromes. Leukemia $\mathbf{3 0}$, 666-673 (2016).

10. Abdel-Wahab, O. et al. ASXL1 mutations promote myeloid transformation through loss of PRC2-mediated gene repression. Cancer Cell 22, 180-193 (2012).

11. Davies, C. et al. Silencing of ASXL1 impairs the granulomonocytic lineage potential of human CD34(+) progenitor cells. Br. J. Haematol. 160, 842-850 (2013).

12. Patnaik, M. M. et al. Prognostic interaction between ASXL1 and TET2 mutations in chronic myelomonocytic leukemia. Blood Cancer J. 6, e385 (2016).

13. Chen, T. C. et al. Dynamics of ASXL1 mutation and other associated genetic alterations during disease progression in patients with primary myelodysplastic syndrome. Blood Cancer J. 4, e177 (2014).

14. Nazha, A. et al. Incorporation of molecular data into the Revised International Prognostic Scoring System in treated patients with myelodysplastic syndromes. Leukemia 30, 2214-2220 (2016). 